\title{
A New Method towards Achieving FES-Induced Movement
}

\author{
M. Ahmed ${ }^{1,2,3^{*}}$, M. S. Huq ${ }^{1,2}$, B. S. K. K. Ibrahim ${ }^{1,2}$
}

${ }^{1}$ Department of Mechatronic and Robotic Engineering, Faculty of Electrical and Electronic Engineering (FKEE)

Universiti Tun Hussein Onn Malaysia (UTHM), 86400 Parit Raja, Batu Pahat, Johor, MALAYSIA.

${ }^{2}$ Advanced Mechatronic Research Group (AdMiRe), Faculty of Electrical and Electronic Engineering (FKEE)

Universiti Tun Hussein Onn Malaysia (UTHM), 86400 Parit Raja, Batu Pahat, Johor, MALAYSIA.

${ }^{3}$ Department of Electrical and Electronics Engineering, Faculty of Engineering and Engineering Technology (FEET) Abubakar Tafawa Balewa University, P. M. B. 0248 Bauchi, Bauchi State, NIGERIA.

Received 3 July 2017; Accepted 7 April 2018, Available online 30 April 2018

\begin{abstract}
Literature has revealed that applications of Functional Electrical Stimulation (FES) for restoration of movements have been yielding promising results in people with impaired neural system. Records indicate rise in subjects with such disabilities without corresponding increase in orthosis devices using FES. The scarce available ones have high cost which could be due strict clinical requirement imposed on such equipment. In order to alleviate the aforementioned challenges an approach was proposing procedure to the system. It suggested improvements for three basic components: plant modelling, re-moudelling the fatigue, spasm and tremor disturbances from the works of Lynch et al., and finally a combined Sliding Mode and Wavelets techniques availing a new control approach for the FES to facilitate safe sit-to-stand movement. There some basic similarities in the sit-to-stand and knee swinging such as; pivot point and muscles stimulated during the processes. The knee joint model proposed by Ferrarin and Pedotti and the disturbances models (fatigue, tremor and spasm) developed by Lynch et al. would be utilized as stated earlier. The procedure was to be conducted phases: plant modeling, disturbances modelling and control system design. Expectations include enhancing subject condition, interactions with others and environment, delivery of health care, self-reliance and reducing health maintenance costs.
\end{abstract}

Keywords: Functional electrical stimulation (FES), control systems, sit-to-stand movement, knee-swinging movement

\section{Introduction}

Electrical stimulations have numerous applications such as pain suppression, enhancing wound healing process, conditioning of skeletal muscles, restoration of vision as well as bladder functions. Functional Electrical Stimulation (FES) is a term which refers to the restoration of motion with the aid of passing electrical signals at appropriate muscles. Loss of the ability for movement usually occurs due to development of disorder in the nervous system usually as a result of accident, disease or damages $[1,2]$.

Currently, combined FES and orthosis are used for movement restoration and rehabilitation as well as for therapy. Control systems for lower limb FES-assisted movements do exist but were a predominantly open loop. The expectation, however, was that shifting to closed loop could enhance both safety and performance for such systems [3-5]. Achieving these could facilitate the emergence of a more FES-aided orthosis which is scarce; imagine even in the United States of America (USA) currently the health department approves only a single device for that purpose [6]. Reports have it that the number of subjects with nervous system ailments kept rising annually $[7,8]$.

The study proposed a new framework for improving the FES induced sit-to-stand (STS) movement. Targetted at improvement towards reaching clinical acceptance. Obtaining the STS model was to be developed from the knee swinging model proposed by Ferrarin and Pedotti [9]. Aimed at simplifying the modelling process due to the nature of the plant. Disturbances to be explored were the Fatigue, spasm and tremor models proposed by Lynch et al. [10-12]. Although disturbance models were comprehensive, there were no available suitable mathematical models. And also, the control scheme is to be developed utilising a combination of sliding mode control and wavelets techniques.

\section{Brief Appraisal of Relevant Literature}

To make things, easier gives a summary of the related literature. It starts with that of FES control schemes for the lower limb, then it looks at the components of the proposed control system and finally the reason for selecting such structure for the system. 


\subsection{Control schemes}

The control methods were for simplicity classified as linear, intelligent and nonlinear techniques. The linear control scheme in this article refers to works that employed linear control theories for solving lower limb FES control problems. Intelligent control scheme refers to the solutions that are obtained using predominantly intelligent methods. For the nonlinear schemes, nonlinear control methods dominate the entire process.

The Linear control schemes were proposed and even put to the test by some researchers, but shortcomings associated with these methods were numerous. Some of which include the need for improvement in sensing (use of better sensors) and the need for re-tuning the control parameters always. Others are stability issues, the difficulty of implementation (most especially in the cases of Linear Quadratic (LQ) and H-infinity control methods and almost all the works are for continuous standing. Lynch and research associates argued that the linear control methods might not be suitable choices for handling FES control [10, 11, 13, 14].

Intelligent control approaches were the most popular for lower limb FES control application. As usual, some studies were simulations while some experimental. Results portrayed fruitfulness that could likely help in passing clinical requirements. Disadvantages include complexity, high tuning duration, retuning required for each subject, high computation time and lack of mathematical model utilisation for ascertaining the stability of the control system. Stability is an important parameter in control systems design. Lack of the complete system mathematical model could make stability analysis very difficult or impossible. Hence, a major shortcoming of the intelligent control schemes when applied [15].

The nonlinear control schemes, although not as popular as the intelligent counterpart but the drawbacks of the linear and intelligent control techniques it a viable option. Additionally, some of the techniques applied to other FES control problems and results are of good impressions. The nonlinear methods could be used for stability analysis making it superior in that regards.

The disadvantages of the linear and intelligent methods give the nonlinear methods upper hand. Perhaps after careful study, it may be integrated with other schemes to yield superb results.

\subsection{Sliding mode control and wavelet networks}

The sliding mode control is a robust nonlinear control technique and is a subdivision of the variable structure. Credited to the works Emelyanov and others in the 1950s from the then Soviet Union; present-day Russia. The technique moves the system very fast along a specified surface to follow the desired trajectory pattern [16].

Wavelet networks technique is a merger of wavelet analysis and artificial neural networks. Early research works on wavelet analysis can be traced back to 1946 through the works of Denis Gabor. Its applications include: for noise removal, compression, and suppression of signals. Also for identifying discontinuity, similarity and changes in signals. Artificial neural networks are numerical models that try to copy the way the nervous system works. Its applied in many areas of endeavours to solve problems successfully. Some of its attributes are the ability to learn, pattern detection, signal processing, detection, and identification. Application of wavelet networks for control system design and system identification is at its infant stage [17-25].

\subsection{Reason Behind the Combination}

Applications of the sliding mode control technique for the lower limb FES control was successful according to literature, and it shows improvement to some extent. The control method has some good characteristics such as insensitivity to external uncertainties and perturbations; the surface could be restricted to follow a particular pattern. As earlier mentioned the shifting along the surface occur very fast, and this could be detrimental to our plant. The ability to eradicate noise as well as the adaptive nature due to inherent intelligence of the wavelet networks could be used to suppress the adverse effect of the high switching rate of the sliding mode without or no significant interference with the mathematical model of the system. Apart from that others are enhanced accuracy, lowering of computing duration as well as the size of memory $[17,25,26]$.

\section{The STS Model}

Obtaining the STS model of paraplegics or subjects with neurological disorders is not an easy task. It was due to the nature of the plant. Additionally, the safety of patients should be guaranteed. Therefore, since the study focused on people with paraplegia, the knee swinging model would be explored. It is because the basic FES induced STS movement can be achieved by stimulating similar muscles (the thigh muscles/quadriceps). The Ferrarin and Pedotti model [9] was chosen to be the basis for the modeling. It was a comprehensive model for the knee joint swinging. Obtained from extensive experimentation. Applying the basic principles of mechanics by considering the necessary forces associated with the STS movement. The model obtained would be validated by comparing with that obtained using principles of robotics. And it would be compared with other existing models. The modelling would be started using the simplest form of scenario. After which it would be generalised to get a model close to that of real humans; that is the more acceptable form. Successfully achieving the model in such a way means revealing a simpler way of obtaining the FES aided STS movement. And it may be extended to other FES induced maneuvres. As well as other forms of neurological disorders. The proposed precedure was as demonstrated by the flowchart by Fig. 1. 


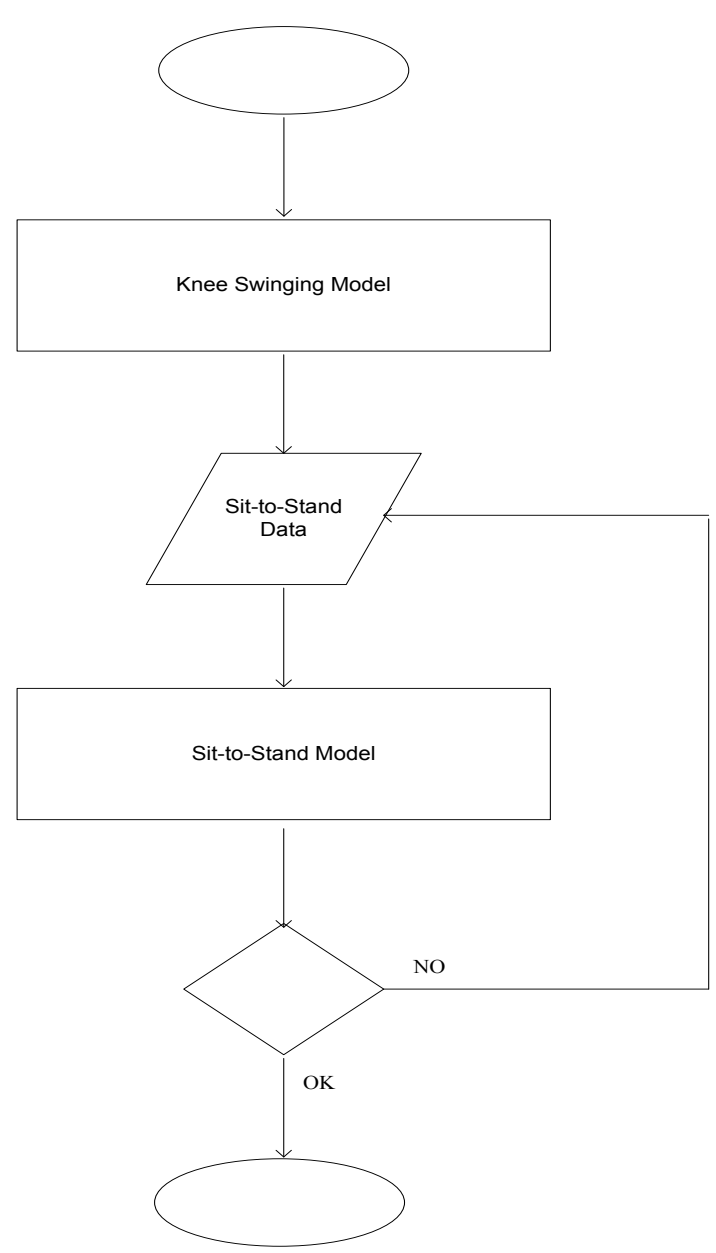

Fig. 1 Modelling the FES induced STS movement flowchart.

\section{Disturbance Models}

The disturbances were fatigue, spasm, and tremor. These are problems seriously associated with neurological disorder patients. Most research works were silent in considering all these disturbances together. Lynch et al. [10-12, 27, 28] reveal that in their research. The disturbance models were from extensive experimentations. The disturbances were very comprehensive as these properties were detailed further into mild, moderate and severe. What hinders the application of the proposed fatigue, spasm and tremor might be its unavailability, although proposed but the mathematical model was not readily available. The effort would be made to obtain the model from the available definitions and pictorial representations of the various disturbance parameters available in their works. Fig. 2 is the flowchart showing how to accomplish the mathematical model of the FES induced STS movement.

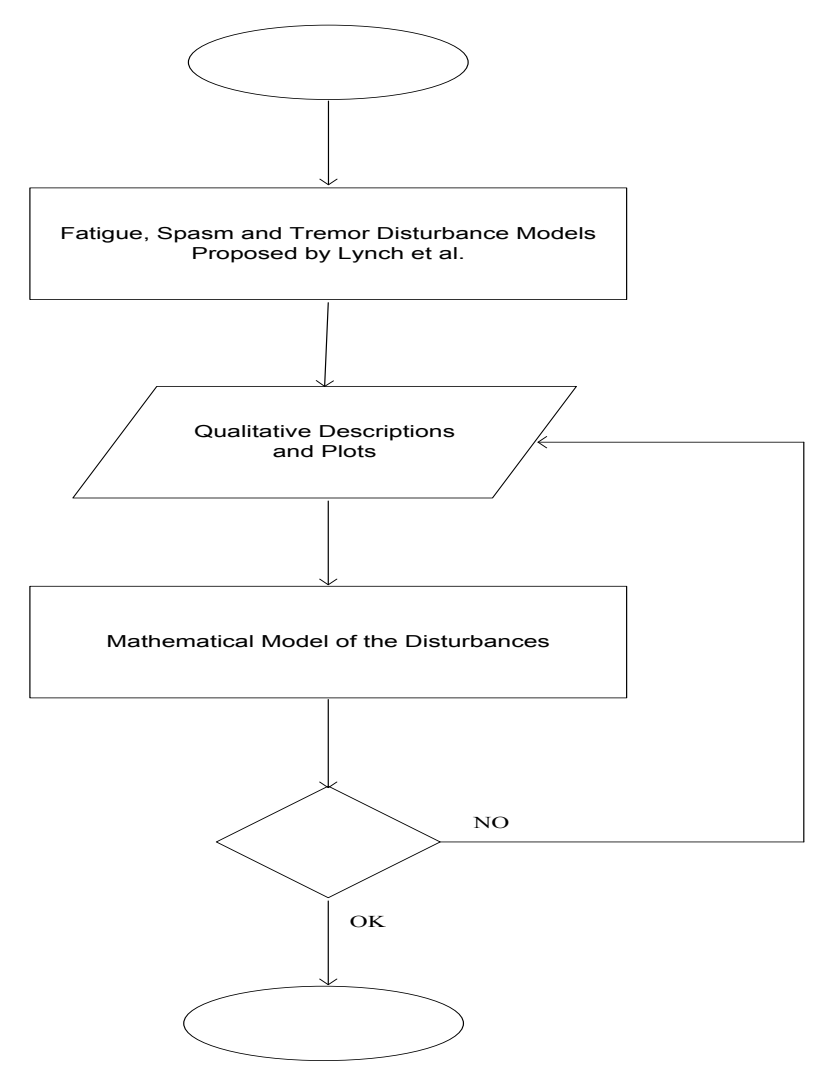

Fig. 2 Disturbance modelling flowchart.

\section{Control System}

The Sliding Mode-Wavelet Networks Controller would be achieved by exploring the robustness capability of the sliding mode technique, noise suppression capability of wavelet networks and the adaptive as well as intellectual abilities of the neural networks. Nature of our plant; that is the neuromuscular being highly nonlinear and associated with so many disturbances. Although the SMC is robust, its associated with high switching rate, that was a treat to fatigue-related the FES activated movements. Noise suppression or smoothening or reducing the rate of switching but still maintaining the control objectives and the neural network capabilities could be explored to improve the adaptation capability of the overall control system which desirable for the application. The sandwich from all indication could result in improving fatigue, tremor, spasm and sensor error suppression. As well as the improved capability for making suitable for different subjects and scenarios. And finally, the control system that can possibly lead to clinical acceptance after implementation. Fig. 3 is the flowchart of the process which could aid in further elaboration of the proposed method. 


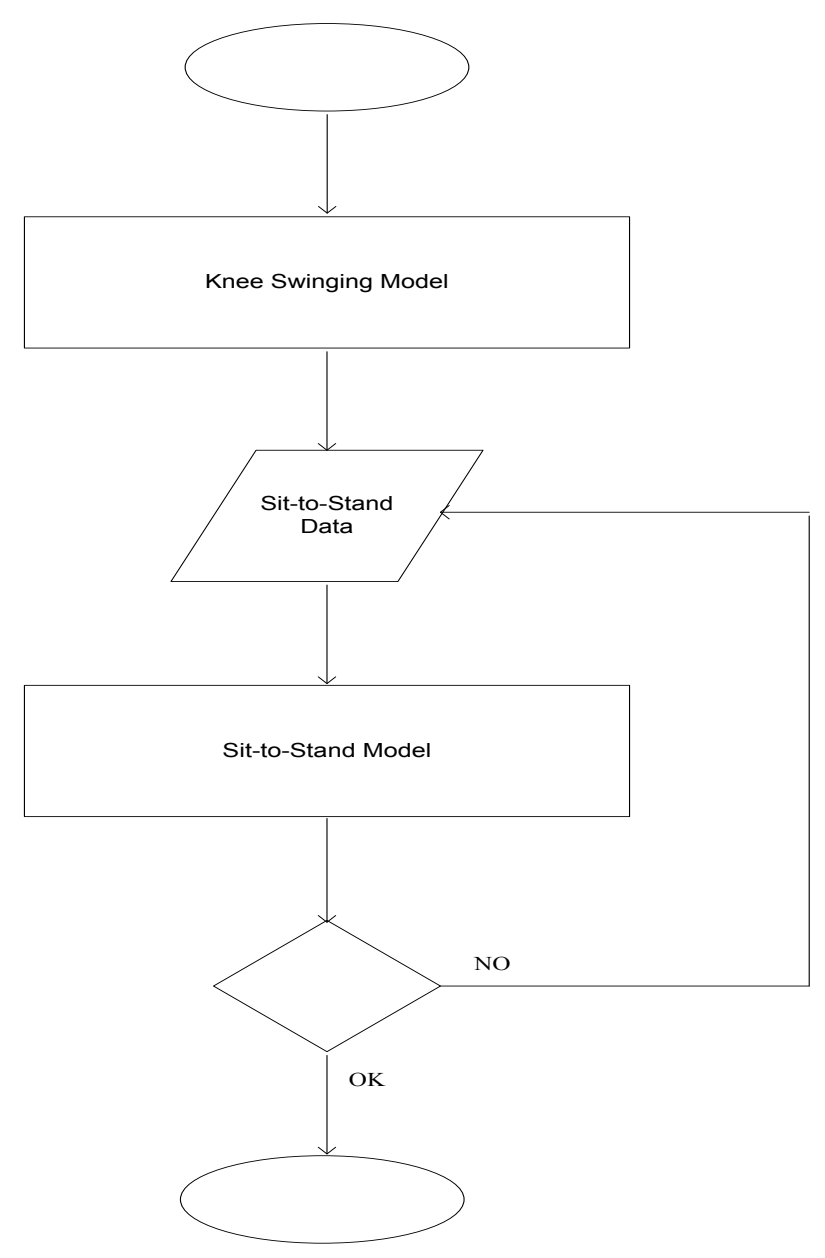

Fig. 3 Control design flowchart.

\section{Expectations}

The work was novel such that it reveals how to obtain STS model from knee swinging model simply. Evaluation of the scheme using fatigue, spasm and tremor as proposed by Lynch and others. And the re-modelling of these properties. And the emergence of a nonlinear control scheme not utilised before which is a combination sliding mode and wavelets techniques. It was expected to be capable of passing clinical trials that may accelerate the production of lower limb FES-assisted orthosis devices. Although the study was intended towards movement restoration in paraplegics, it is also applicable to subjects with other forms of nervous system disruptions. It can also be used for gait restoration and for remedy purposes. Others include: lowering maintenance costs maintenance and improvement of physical and mental health, making the patients more self-reliance and sociable [29, 30].

\section{Summary}

Earlier research works have clearly indicated that FES technique is propitious for not only restoration of movements, but also for remedy and gait management in subjects with nervous system malfunctions. The figure of such patients kept becoming higher with no similar growth in FES-assistive devices, which might be due to inability to pass clinical standards. The new approach presented showed steps that could lead to the system improvement. It tried to avail enhanced modelling steps, and verification could be made after everything is well established. Presenting viable stages to obtain the mathematical model of the disturbances by Lynch et al. And finally, a revealing new way of controlling the system utilising Sliding mode and Wavelets methods. Achieving the objectives using the proposed procedure could lead to enhanced system. And it is expected to be efficient, automated and able to pass clinical tests.

\section{References}

[1] W. K. Durfee, "Gait restoration by functional electrical stimulation," in Climbing and Walking Robots, ed: Springer, 2006, pp. 19-26.

[2] G. P. Braz, M. Russold, and G. M. Davis, "Functional electrical stimulation control of standing and stepping after spinal cord injury: a review of technical characteristics," Neuromodulation: Technology at the Neural Interface, vol. 12, pp. 180190, 2009.

[3] Q. Zhang, M. Hayashibe, P. Fraisse, and D. Guiraud, "FES-induced torque prediction with evoked EMG sensing for muscle fatigue tracking," Mechatronics, IEEE/ASME Transactions on, vol. 16, pp. 816-826, 2011.

[4] Q. Zhang, M. Hayashibe, and C. Azevedo-Coste, "Evoked electromyography-based closed-loop torque control in functional electrical stimulation," IEEE Transactions on Biomedical Engineering,, vol. 60, pp. 2299-2307, 2013.

[5] D. Simon, D. Andreu, and S. Lafnoune, "Real-time Simulation for a Functional Electrical Stimulation system validation," in 10th National Conference on Control Architectures of Robots (CAR) 2015, Lyon, France, 2015.

[6] CMS. (2015, November 24). Decision Memo for Neuromuscular Electrical Stimulation (NMES) for Spinal Cord Injury (CAG-00153R). Available: www.cmsgov/medicare-coverage-database/details/ Decision Memo for Neuromuscular Electrical Stimulation (NMES) for Spinal Cord Injury (CAG00153R)

[7] W. H. Organization, Neurological disorders: public health challenges: World Health Organization, 2006.

[8] A. Officer, T. Shakespeare, P. von Groote, J. Bickenbach, and W. H. Organization, International perspectives on spinal cord injury: World Health Organization, 2013.

[9] M. Ferrarin and A. Pedotti, "The relationship between electrical stimulus and joint torque: A dynamic model," IEEE transactions on rehabilitation engineering, vol. 8, pp. 342-352, 2000.

[10] C. L. Lynch, G. M. Graham, and M. R. Popovic, "A generic model of real-world non-ideal behaviour of FES-induced muscle contractions: simulation tool," 
Journal of neural engineering, vol. 8, p. 046034, 2011.

[11] C. L. Lynch, G. M. Graham, and M. R. Popovic, "Including nonideal behavior in simulations of functional electrical stimulation applications," Artificial organs, vol. 35, pp. 267-269, 2011.

[12] C. Lynch, "Closed-Loop Control of Electrically Stimulated Skeletal Muscle Contractions," PhD Doctoral dissertation, 2011.

[13] C. L. Lynch, "Closed-Loop Control of Electrically Stimulated Skeletal Muscle Contractions," PhD, University of Toronto, 2011.

[14] C. Lynch and M. Popovic, "Closed-loop control for FES: Past work and future directions," in 10th Annual Conference of the International FES Society, 2005, pp. 2-4.

[15] M. Huq and M. Tokhi, "Genetic algorithms based approach for designing spring brake orthosis-Part II: Control of FES induced movement," Applied Bionics and Biomechanics, vol. 9, pp. 317-331, 2012.

[16] J. Liu and X. Wang, Advanced sliding mode control for mechanical systems: Springer, 2012.

[17]A. K. Alexandridis and A. D. Zapranis, "Wavelet neural networks: A practical guide," Neural Networks, vol. 42, pp. 1-27, 2013.

[18] S. A. Billings and H.-L. Wei, "A new class of wavelet networks for nonlinear system identification," Neural Networks, IEEE Transactions on, vol. 16, pp. 862-874, 2005.

[19] M. Misiti, Y. Misiti, G. Oppenheim, and J.-M. Poggi, "Wavelet toolbox for use with Matlab, the MathWorks," Natick, MA, 1996.

[20]I. W. Selesnick, "Wavelet Transforms-A Quick Study," Physics Today magazine, 2007.

[21] J. C. Goswami and A. K. Chan, Fundamentals of wavelets: theory, algorithms, and applications vol. 233: John Wiley \& Sons, 2011.

[22]A. K. Mutlag, "DYNAMIC SYSTEM IDENTIFICATION USING TIME-DELAY FEEDFORWARD NEURAL NETWORKS:
APPLICATION TO DC MOTOR," Diyala Journal of Engineering Sciences, vol. 3, pp. 65-79, 2010.

[23] O. Jovanović, "Identification of dynamic system using neural network," The Scientific Journal FACTA UNIVERSITATIS Series: Architecture and Civil Engineering, vol. 31, pp. 525-532, 1997.

[24] J. Liu, Radial Basis Function (RBF) neural network control for mechanical systems: design, analysis and Matlab simulation: Springer Science \& Business Media, 2013.

[25] S.-T. Tzeng, "Design of fuzzy wavelet neural networks using the GA approach for function approximation and system identification," Fuzzy Sets and Systems, vol. 161, pp. 2585-2596, 2010.

[26] D. M. Salih, S. B. M. Noor, M. Hamiruce Merhaban, and R. M. Kamil, "Wavelet Network: Online Sequential Extreme Learning Machine for Nonlinear Dynamic Systems Identification," Advances in Artificial Intelligence, vol. 2015, 2015.

[27] C. L. Lynch and M. R. Popovic, "A stochastic model of knee angle in response to electrical stimulation of the quadriceps and hamstrings muscles," Artificial organs, vol. 35, pp. 1169-1174, 2011.

[28] C. L. Lynch and M. R. Popovic, "A comparison of closed-loop control algorithms for regulating electrically stimulated knee movements in individuals with spinal cord injury," IEEE Transactions on Neural Systems and Rehabilitation Engineering, vol. 20, pp. 539-548, 2012.

[29]P. Taylor, L. Humphreys, and I. Swain, "The longterm cost-effectiveness of the use of functional electrical stimulation for the correction of dropped foot due to upper motor neuron lesion," Journal of rehabilitation medicine, vol. 45, pp. 154-160, 2013.

[30]N. Byl, W. Zhang, S. Coo, and M. Tomizuka, "Clinical impact of gait training enhanced with visual kinematic biofeedback: Patients with parkinson's disease and patients stable post stroke," Neuropsychologia, vol. 79, pp. 332-343, 2015. 